\title{
De la loi Huriet à la loi Jardé
}

\section{From Huriet law to Jardé law}

\author{
F. Lemaire $\cdot$ M. Matei \\ (C) SRLF et Springer-Verlag France 2012
}

Le 6 mars 2012, la loi relative aux « recherches impliquant la personne humaine », dite loi Jardé, promulguée la veille, était publiée au Journal Officiel [1]. Après plusieurs révisions, dont la plus importante avait eu lieu en août 2004, la loi Huriet (1988) avait été à nouveau remise en chantier. Ainsi, par un processus d'évolution quasi itératif, la législation sur la recherche s'est-elle progressivement adaptée à la totalité de la recherche « impliquant la personne », alors qu'à l'origine elle avait été conçue pour encadrer la recherche sur le médicament. La loi Huriet avait en effet été rédigée au départ par des pharmacologues [2], qui entendaient autoriser en France les recherches de phase 1 portant sur le médicament, illégales jusque-là [3]. Même si ses dispositions avaient été rapidement étendues à l'ensemble de la recherche interventionnelle, elles n'étaient véritablement adaptées qu'aux recherches portant sur le médicament. Et c'est sous la pression des investigateurs " académiques » que des dispositions correspondant mieux à leurs recherches (en chirurgie, pédiatrie, réanimation, médecine aiguë, épidémiologie...) [4,5] ont pu être adoptées, non sans la forte résistance des nombreux « gardiens du dogme » [6]. La loi relative à la politique de santé publique (2004) avait supprimé l'inutile et trompeuse distinction entre les recherches avec et sans bénéfice individuel direct, introduit une procédure allégée pour les " recherches portant sur les soins courants » [7], renforcé les procédures d'autorisation et de contrôle, amplifié les missions et responsabilités des promoteurs.

Cependant, de nombreuses difficultés subsistaient, qui pénalisaient surtout les investigateurs du champ académique. Les dispositions relatives à la recherche portant sur les soins courants étaient insuffisamment allégées. Les recherches non interventionnelles restaient hors champ, entraînant de plus en plus fréquemment des restrictions à la

\footnotetext{
F. Lemaire $(\bowtie) \cdot$ M. Matei

Département de la recherche clinique et du développement

de l'Assistance Publique, Hôpitaux de Paris (AP-HP),

Paris, France

e-mail : francois.lemaire@sls.aphp.fr
}

publication dans les revues les plus prestigieuses. Les dispositions concernant les collections biologiques étaient inapplicables. Les recherches en génétique portant sur des échantillons biologiques issus de patients décédés et qui n'avaient pas consenti de leur vivant restaient interdites. Une nouvelle révision était devenue inévitable.

En étendant le champ de la loi aux recherches non interventionnelles, dites aussi observationnelles, la loi du 5 mars 2012 unifie en effet les différentes catégories de recherche existantes dans un ensemble unique : la « recherche impliquant la personne humaine ». Un socle réglementaire commun impose la soumission de tous les projets de recherche à un Comité de protection des personnes (CPP) et la désignation d'un promoteur.

La loi identifie désormais au sein de cet ensemble trois catégories de recherches selon le niveau de risque encouru par la personne qui s'y prête : les recherches interventionnelles avec risque supérieur au risque minime, celles qui ne comportent qu'un risque minime et qui ne portent pas sur un médicament et les recherches non interventionnelles. Dans chacune de ces trois catégories, le niveau de contrainte réglementaire et les conditions de recueil du consentement sont différents et modulés en fonction du risque. Au-delà, une adaptation fine des modalités de recueil du consentement en fonction de certaines recherches particulières a été inscrite dans la loi, notamment dans le cas des recherches avec risque minime en pédiatrie ou lorsque la méthodologie de l'essai ne permet pas le recueil d'un consentement exprès (clusters, recherche épidémiologique interventionnelle). En ce qui concerne les recherches en situation d'urgence extrême, qualifiée de " vitale immédiate » dans la loi, il sera désormais possible de démarrer la recherche sans l'autorisation préalable de la personne de confiance, de la famille ou des proches, même s'ils sont présents. En outre, les conditions du « consentement de poursuite » sont harmonisées dans les deux catégories voisines que sont la recherche en situation d'urgence et lorsque la personne est " hors d'état » de consentir, un contentieux récent jugé au tribunal de grande instance de Paris [8] ayant révélé les ambiguités et les difficultés d'interprétation des dispositions en vigueur à cet égard. 
De grands changements vont également concerner les CPP. Ceux-ci voient leur rôle diversifié et amplifié de façon notable. En outre, la loi a créé une commission nationale placée auprès du ministre de la Santé afin d'harmoniser le fonctionnement de ces comités, jugé communément très inégal, et de mieux les coordonner.

Enfin, de nombreuses améliorations ponctuelles vont être apportées à certaines dispositions inapplicables aujourd'hui, telles la mauvaise cohérence entre la loi bioéthique et la loi relative à la politique de santé publique concernant les collections de produits biologiques, ou le financement du médicament expérimental lorsqu'il a une autorisation de mise sur le marché et qu'il est utilisé dans un essai à « finalité non commerciale $\gg$.

La loi ne sera applicable qu'après la parution des principaux décrets d'application, sans doute au début de l'année 2013. Au paradigme établi en 1988 d'une recherche définie comme l'atteinte au corps des personnes qui s'y prêtent dans une finalité autre que thérapeutique, la loi de mars 2012 substitue la notion de recherche « impliquant la personne », dans une finalité de production de connaissance. L'élargissement du champ d'application de la loi à des recherches moins « invasives » (recherches avec risque minime et observationnelles) a pour corollaire l'adaptation de ses dispositions aux risques et contraintes ajoutés par la recherche. Le concept de procédures « risk adjusted » est d'ailleurs aujourd'hui à l'étude dans la plupart des autres pays où la recherche est développée, l’Union européenne [9], les États-Unis [10] et le Royaume-Uni [11].

Conflit d'intérêt : les auteurs déclarent ne pas avoir de conflit d'intérêt.

\section{Références}

1. loi du 5 mars 2012 : http://www.legifrance.gouv.fr/affichCode Article.do;jsessionid=16FBF66168900EE5C4E0FCD5514460B8. tpdjo14v_2?idArticle $=$ LEGIARTI000025449446\&cidTexte $=$ LEGITEXT000006072665\& categorieLien $=$ id\&dateTexte $=22220222$ (accès le 1205 2012)

2. Jaillon P, Demarez JP (2008) L'histoire de la genèse de la loi Huriet-Sérusclat de décembre 1988. Med Sci (Paris) 24:323-7

3. Rapport fait au nom de la Commission des affaires sociales du Sénat par Claude Huriet, 11 octobre 1988

4. Lemaire F (2001) Révision de la loi du 20 décembre 1988 : plateforme commune de propositions émanant des sociétés savantes, organismes et associations de malades. Reanimation 10:435-8

5. Lemaire F, Ravoire S, Golinelli D (2008) Recherche non interventionnelle : définition, aspects réglementaires, difficultés et propositions. Thérapie 63:97-101

6. Gomez M (2004) Controverse autour de futurs essais thérapeutiques. La Croix, 24 avril 2004

7. Matei M, Thalabard JC, Laude A, et al (2011) Recherche portant sur les soins courants : évaluation des critères de qualification proposés par le dispositif législatif et réglementaire en vigueur. Presse Med 40:e189-e196

8. Matei M (2011) La recherche en situation d'urgence : la famille doit-elle consentir ? La Gazette du Palais janvier pp 23-7

9. Revision of the clinical trial directive 2001/20/EC. Concept paper submitted for public consultation. http://www.mhra.gov.uk/home/ groups/1-ctu/documents/websiteresources/con111784.pdf (accès le 1405 2012)

10. ANPRM for Revision to Common Rule: Information Related to Advanced Notice of Proposed Rulemaking (ANPRM) for Revisions to the Common Rule: http://www.gpo.gov/fdsys/pkg/FR2011-09-01/pdf/2011-22341.pdf

11. MRC/DH/MHRA Joint Project (accès le 14-05-2012) Riskadapted Approaches to the Management of Clinical Trials of Investigational Medicinal Products: http://www.mhra.gov.uk/ home/groups/1-ctu/documents/websiteresources/con111784.pdf (accès le 14-05-2012) 